\title{
PREVALÊNCIA DA POLIFARMÁCIA NOS IDOSOS DE UMA UNIDADE BÁSICA DE SAÚDE NO ESTADO DE MINAS GERAIS
}

\author{
PREVALENCE OF POLYPHARMACY IN THE ELDERLY OF A BASIC HEALTH UNIT \\ IN THE STATE OF MINAS GERAIS
}

\author{
Priscila Assis da Silveira ${ }^{a^{*}}$, Samuel Campos Silva ${ }^{b^{*}}$, Karine Siqueira Cabral Rocha $^{c^{\star}}$ \\ Centro Universitário de Patos de Minas (UNIPAM) ${ }^{a}$, Centro Universitário de Patos de Minas (UNIPAM) ${ }^{\mathrm{b}}$, Centro Universitário de Patos de Minas
} (UNIPAM)

\section{RESUMO}

Introdução: Sabe-se que o uso excessivo de medicamentos é na atualidade um grande problema de saúde pública, que pode repercutir negativamente na vida dos indivíduos, principalmente na população idosa. De acordo com a Organização Mundial da Saúde a população idosa é aquela a partir de 60 anos de idade. Polifarmácia é a utilização de pelo menos cinco medicamentos de uso contínuo. Objetivo: Analisar a prevalência da polifarmácia em idosos de uma unidade básica de saúde (UBS), correlacionando com o gênero e a faixa etária. Metodologia: Trata-se de um estudo observacional retrospectivo dos idosos de uma UBS, no município de Patos de Minas, cadastrados no Sistema Único de Saúde. Resultados: De acordo com as análises, dos 282 participantes, 40,4\% (114) encontravam-se em polifarmácia, sendo que 45,2\% (84) eram mulheres e apenas $31,25 \%$ (30) eram homens. A pesquisa também mostrou uma relação significativa entre polifarmácia e faixa etária, onde 31,09\% dos idosos entre 60 e 69 anos encontravam-se em polifarmácia, 46,49\% dos entre 70 e 79 anos e 48,97\% dos entre 80 e 89 anos. A prevalência encontrada de polifarmácia em idosos no presente estudo foi maior que os dados encontrados na literatura. Conclusão: Os dados analisados evidenciam a relação entre o gênero feminino com a polifarmácia, ou seja, ser idosa no estudo constituiu um fator de risco para polifarmácia.

Palavras-chave: Polimedicação; idoso; medicações.

\begin{abstract}
Introduction: It is known that the excessive use of medications is currently an immense public health problem, which can negatively affect the life of individuals, especially in the elderly population. According to the World Health Organization the elderly population comprehends those who are 60 years of age or older. Polypharmacy is the use of at least five medications of continuous use. Objective: The present study had the objective of analyzing the prevalence of polypharmacy in the elderly of a basic health unit (UBS), correlating with gender and age range. Method: This is a retrospective observational study of the elderly of a UBS in the city of Patos de Minas, registered in the Unified Health System. Results: According to the analysis, of the 282 participants, $40.4 \%$ (114) were in polypharmacy, $45.2 \%$ (84) of whom were women and only $31.25 \%$ (30) were men. The research also showed significant relation between polypharmacy and the age range, in which $31.09 \%$ of the group of 60 to 69 years of age were in polypharmacy, $46.49 \%$ of those between 70 and 79 and $48.97 \%$ of those between 80 and 89 years. The prevalence of polypharmacy in the elderly found in this study was higher than data found in literature. Conclusions: The results show an association between the female gender and polypharmacy, that is, being an older woman in the study was a risk factor for polypharmacy.
\end{abstract}

Keywords: Polypharmacy; elderly; medications. 


\section{INTRODUÇÃO}

De acordo com a Organização Mundial da Saúde (OMS) a população idosa é aquela a partir de 60 anos de idade, porém esse limite é válido apenas para países em desenvolvimento, elevando para 65 anos de idade nos países desenvolvidos. Já uma população envelhecida é um estado quando a proporção de pessoas com 60 anos ou mais atinge $7 \%$ com tendência a crescer. De acordo com o Censo Demográfico de 2010, os brasileiros com 60 anos ou mais já somavam 20.590.599 indivíduos, representando $10,8 \%$ da população total, logo, não se pode mais dizer que o Brasil seja um país jovem. As mudanças demográficas e epidemiológicas possuem significativa relevância para os gastos públicos com saúde e previdência. A modificação da longevidade da população está alterando o perfil epidemiológico do país, com um aumento da mortalidade por doenças crônicas não transmissíveis em detrimento das doenças infecto-parasitárias ${ }^{1}$..

Segundo o censo demográfico de $2010^{1}$, a população brasileira de hoje é de 190.755 .199 milhões de pessoas. $\mathrm{O}$ contingente de pessoas idosas é de 20.590.599 milhões, ou seja, aproximadamente $10,8 \%$ da população total. Desses, 55,5 \% (11.434.487) são mulheres e 44,5\% (9.156.112) são homens. De acordo com as projeções do Instituto Brasileiro de Geografia e Estatística (IBGE), em 2022 o Brasil contará com uma população de aproximadamente 209,4 milhões de pessoas e a pirâmide etária não mais fará jus a esse nome, devido à continuada tendência ao envelhecimento populacional.

Polifarmácia é a utilização de pelo menos cinco medicamentos de uso contínuo ${ }^{2}$. Existem diferentes recomendações para defini-la, sendo que alguns autores consideram a polifarmácia apenas a relacionada com os medicamentos utilizados sem indicação ou em doses exageradas. A polifarmácia constitui-se uma prática frequente entre os idosos, sua prevalência em estudos brasileiros varia de 5 a $27 \%^{3,4}$.

Destaca-se o fato de que o desenvolvimento tecnológico e as estratégias farmacológicas de promoção de saúde têm contribuído para o aumento da utilização de medicamentos nas últimas décadas, além do crescimento da indústria farmacêutica e o marketing dos medicamentos. Somando ao fato de que os idosos possuem peculiaridades fisiológicas, além de múltiplas condições patológicas que eleva a prescrição de fármacos. No Brasil, cerca de $70 \%$ dos idosos apresenta pelo menos uma enfermidade crônica, precisando de tratamento farmacológico e uso regular dos medicamentos. Este contexto contribui para que os idosos representem uma significativa parcela dos consumidores de medicamentos e muitas vezes, de modo conjunto - a polifarmácia ${ }^{5}$.

Alguns fatores estão associados à polifarmácia: sexo feminino, idade $\geq 80$ anos, autoavaliação de saúde regular, doenças crônicas e número de consultas médicas no último ano. $\mathrm{O}$ crescimento da indústria farmacêutica e o marketing de medicamentos também podem contribuir para o aumento das prescrições pelos profissionais de saúde, propiciando o uso de múltiplos medicamentos pelos idosos. Uma das razões que poderia explicar a duplicidade de prescrição é que a maioria dos idosos tem dificuldade de lembrar qual medicamento utiliza, havendo a possibilidade de um especialista prescrever um fármaco com a mesma ação de um já utilizado pelo paciente ${ }^{6}$.

Outro fator a ser considerado é a reação adversa que, em idosos, muitas vezes não considera que o sintoma relatado possa ser reação adversa de um fármaco em uso. Isto consequentemente resultará na prescrição desnecessária de outro fármaco e não na substituição ou alteração, aumentando a polifarmácia nesses pacientes 7 .

A polifarmácia nos idosos poderá comprometer a morbimortalidade e a qualidade de vida destes indivíduos, sendo uma das principais consequências o aumento do risco das reações adversas aos medicamentos, que compreendem um grupo diversificado de sintomas e enfermidades de vários sistemas ${ }^{5}$.

Do ponto de vista farmacológico, a polifarmácia proporciona um aumento da chance de ocorrência de interações medicamentosas. Devido ao sinergismo ou antagonismo de múltiplos fármacos com interferência no metabolismo hepático e no mecanismo de excreção pode-se ocorrer desde a potencialização de efeitos adversos até a diminuição da ação dos fármacos. Neste contexto, o presente estudo teve como objetivo analisar a prevalência da polifarmácia em idosos de uma unidade básica de saúde, correlacionando com a 
faixa etária e o gênero. O estudo se justificou uma vez que a polifarmácia associa-se a um maior número de reações adversas aos medicamentos, interações medicamentosas, dificuldades na adesão ao tratamento e aumento dos custos da assistência à saúde, além de que a múltipla medicação é um fenômeno atual e relevante, especialmente em populações idosas.

\section{METODOLOGIA}

O município escolhido para a realizada da presente pesquisa foi Patos de Minas, que está localizado no Alto Paranaíba a $415 \mathrm{~km}$ de Belo Horizonte, capital do Estado de Minas Gerais. Segundo o Censo de 2010 do $\mathrm{IBGE}^{1}$ dos 138.710 habitantes, $127.724(92,08 \%)$ vivem na cidade, enquanto 10.986 (7,92\%) são moradores do campo. Com uma área de $3.189,771 \mathrm{~km}^{2}$, a densidade demográfica do município é de $43,49 \mathrm{hab} / \mathrm{km}^{2}$. Dos 138.710 habitantes, 16.359 são idosos, totalizando $11,8 \%$ dos habitantes.

A Unidade Básica de Saúde pesquisada é formada por seis micro áreas e abrange cerca de 3600 pessoas, destas, aproximadamente 500 são idosos $(14,5 \%)$.

Trata-se de um estudo observacional retrospectivo dos idosos de uma Unidade Básica de Saúde (UBS), no município de Patos de Minas, cadastrados no Sistema Único de Saúde (SUS).

Foram incluídos no estudo todos os pacientes com idade igual ou superior a 60 anos, cadastrados no SUS e pertencentes à UBS. Somente foram incluídos como idoso em polifarmácia os idosos em uso de cinco ou mais medicamentos no período de janeiro de 2016 até junho de 2017, quando finalizou a coleta. Foram excluídos aqueles pacientes que apresentavam dados incompletos em seus prontuários e aqueles cujos prontuários constaram consultas com datas anteriores à janeiro de 2016. Os prontuários foram analisados somente no período de abril a junho de 2017, foram avaliados como um todo, não só o período em questão.

Foi realizada uma reunião com os agentes de saúde da área estudada para saber a quantidade aproximada de idosos a ser analisados, com isso chegou à conclusão de aproximadamente 500 idosos pertencentes aquela unidade básica de saúde.
A Unidade Básica de Saúde foi escolhida para o projeto devido a expressiva densidade demográfica de idosos nessa região, estão cadastrados na Unidade de Saúde escolhida um total de aproximadamente 500 idosos numa população de 3.447 pessoas cadastradas, totalizando uma porcentagem de $14,5 \%$ de idosos. No município de Patos de Minas, a população total era de 138.710 habitantes com 16.359 idosos, totalizando $11,8 \%$ de idosos. A Unidade foi escolhida também devido à aceitação da pesquisa e ao expressivo número de idosos que ultrapassa a porcentagem da população geral do município.

Foi realizada uma análise dos prontuários $\mathrm{e}$ cada idoso foi colocado em uma planilha, seus dados foram classificados quanto ao número da família, gênero, data de nascimento, número de medicamentos de uso contínuo e data da última consulta; com o intuito de verificar a prevalência da polifarmácia naquela equipe.

A prevalência mede a proporção de pessoas numa dada população que apresenta uma específica doença ou atributo, num determinado tempo no espaço. No cálculo da prevalência do projeto o numerador abrange o número de idosos que estão em polifarmácia num determinado período (01 de abril a 31 de junho de 2017). Por sua vez, o denominador é a população total de idosos no mesmo período. A prevalência pode ser expressa da seguinte forma: $\mathrm{P}=$ número de idosos em polifarmácia / número total de idosos

$\mathrm{O}$ instrumento utilizado foi uma tabela com as seguintes variáveis: número da família, gênero, data de nascimento, número de medicamentos de uso contínuo, data da última consulta.

Para o desenvolvimento deste estudo, o projeto foi submetido ao Comitê de Ética em Pesquisa do Centro Universitário de Patos de Minas. Esta pesquisa seguiu os princípios éticos presentes na Resolução no 466, de 12 de dezembro de 2012 do Conselho Nacional de Saúde. O projeto foi submetido ao Comitê de Ética e Pesquisa, sendo aprovado com o parecer número 1.946.930.

Para as análises estatísticas foi utilizado o software Statistical Package for Social Sciences (SPSS), versão 20.0 para Windows. As frequências das respostas foram submetidas a análises comparativas entre duas proporções pelo teste de Qui-quadrado $(\chi 2)$. Todos os resultados foram consi- 
derados significativos a um nível de significância de $\mathrm{p}<0,05$.

\section{RESULTADO E DISCUSSÃO}

$\mathrm{Na}$ análise realizada com os prontuários, dentre os 500 que foram escolhidos para participar da pesquisa, foi necessária a exclusão de 186 devido ao fato de a última consulta ter acontecido antes de janeiro de 2016. Também foram excluídos 32 prontuários por estarem incompletos. Portanto, restaram 282 prontuários de idosos para análise da pesquisa.

Em relação aos dados sociodemográficos é possível perceber que a maior parte da população idosa pesquisada é do sexo feminino (65,9\%), está na faixa etária entre 60 a 69 anos $(42,1 \%)$ e participou de consulta médica pela última vez no período de julho e dezembro de 2016 (62,7\%). Abaixo estão os gráficos que demonstram esses resultados.

Gráfico 1: Gênero dos idosos. Patos de Minas (MG-Brasil), 2017.

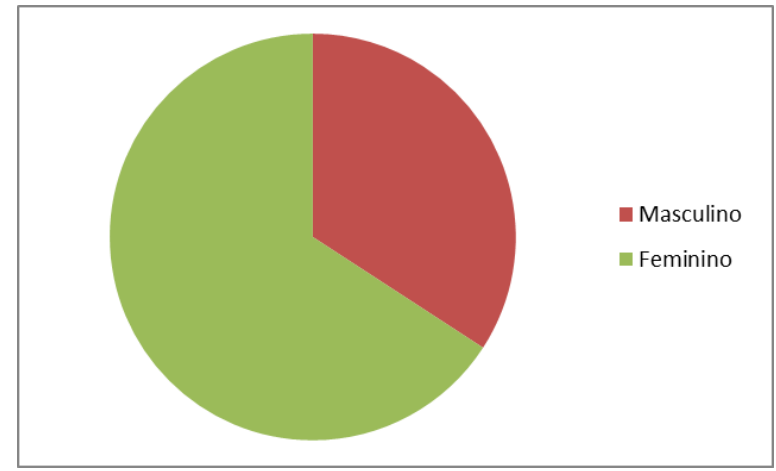

Fonte: autoria própria.

Gráfico 2: Faixa etária dos idosos. Patos de Minas (MG-Brasil), 2017.

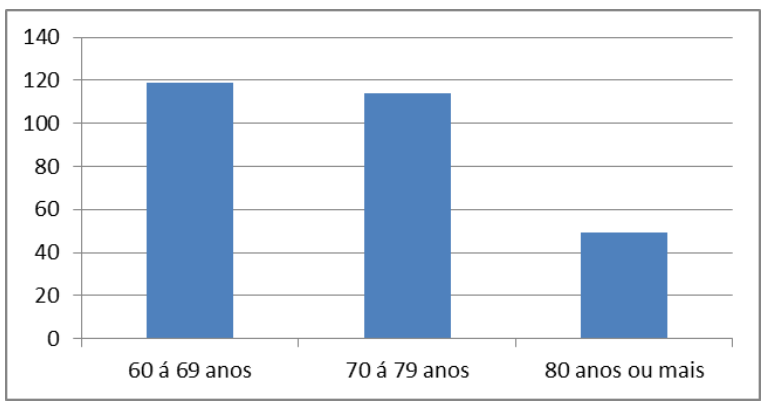

Fonte: autoria própria.
Gráfico 3: Período da última consulta dos idosos. Patos de Minas (MG-Brasil), 2017.

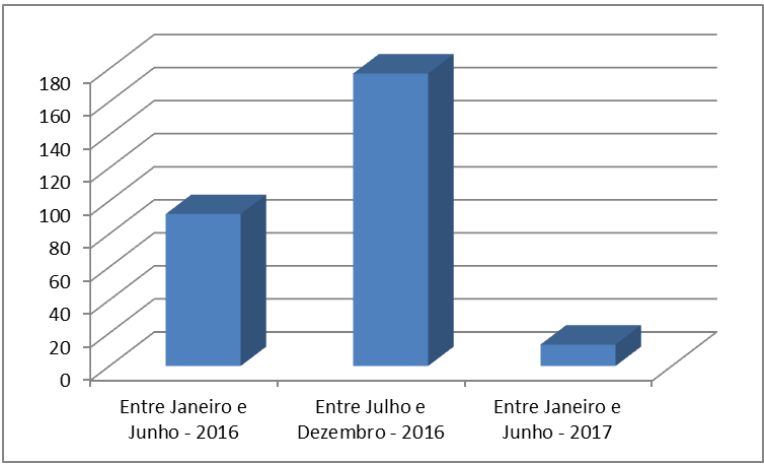

Fonte: autoria própria.

De acordo com as análises, dos 282 participantes, $114(40,4 \%)$ encontravam-se em polifarmácia, com a utilização de 5 ou mais medicamentos de uso contínuo. Ainda nesta mesma análise foi possível observar que dentre os idosos analisados, $20,6 \%$ utilizavam quatro medicamentos, $13,5 \%$ faziam uso de três fármacos, $14,2 \%$ utilizavam dois medicamentos, $7,1 \%$ utilizavam um fármaco $\mathrm{e}$ apenas $4,2 \%$ não faziam uso de nenhum medicamento, como demonstra o gráfico quatro a seguir.

Gráfico 4: Quantidade de idosos x número de medicamentos. Patos de Minas (MG-Brasil), 2017.

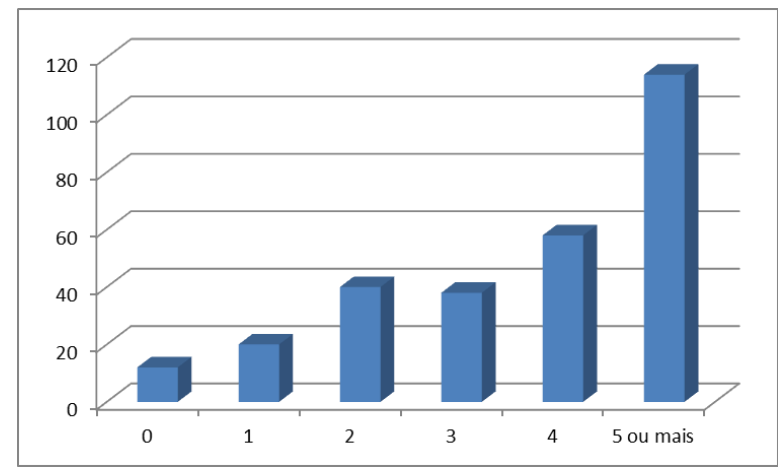

Fonte: autoria própria.

De acordo com o resultado obtido fica nítida a elevada prevalência da polifarmácia na população idosa estudada. Resultados semelhantes foram encontrados por Carvalho (2007) $)^{4}$ ao analisar a polifarmácia em idosos de São Paulo, e encontrar $31,5 \%$ de prevalência. É interessante destacar que, mesmo o Brasil sendo considerado um país subdesenvolvido, os resultados obtidos encaixam-se 
nos valores estimados de países desenvolvidos, onde 20 a $40 \%$ dos idosos nesses países utilizam múltiplos medicamentos associados ${ }^{8}$.

$\mathrm{Na}$ análise da relação entre a polifarmácia e o gênero dos idosos pesquisados, foi obtido o resultado de que das 183 mulheres participantes da pesquisa, 84 delas, ou seja, $45,2 \%$ se encontravam em polifarmácia. Já dentre os 96 homens participantes, apenas 30 deles, ou 31,25\% encontrava-se em polifarmácia.

A análise estatística permitiu concluir que a prevalência da polifarmácia tem relação significativa com o gênero feminino, com $\mathrm{p}=0,01205$. Ou seja, o gênero feminino se constituiu fator de risco para a prevalência da polifarmácia. Ramos et al., $(2016)^{9}$ também encontrou relação significativa entre a polifarmácia em idosos e o sexo feminino. Outro estudo que também obteve resultados semelhantes foi o de Almeida et al., $(2017)^{10}$, que percebeu níveis significativamente elevados de polifarmácia nas mulheres.

Aqui é possível levantar a hipótese de que o resultado foi influenciado devido ao fato de a maior parte da população estudada $65,95 \%$ ser do sexo feminino. Ou seja, aqui fica a dúvida se ser do sexo feminino é realmente um risco para a polifarmácia, ou se seria porque as mulheres são a maior parte da população que utiliza a unidade básica para melhor acompanhamento da saúde, visto que a saúde do homem ainda é um alvo do Ministério da Saúde devido ao fato deles, em sua maioria, não procurarem auxílio médico para exames de rotina e prevenção de doenças. Abaixo estão os gráficos referentes à essa análise.

Gráfico 5:Gênero x Polifarmácia. Patos de Minas (MG-Brasil), 2017.

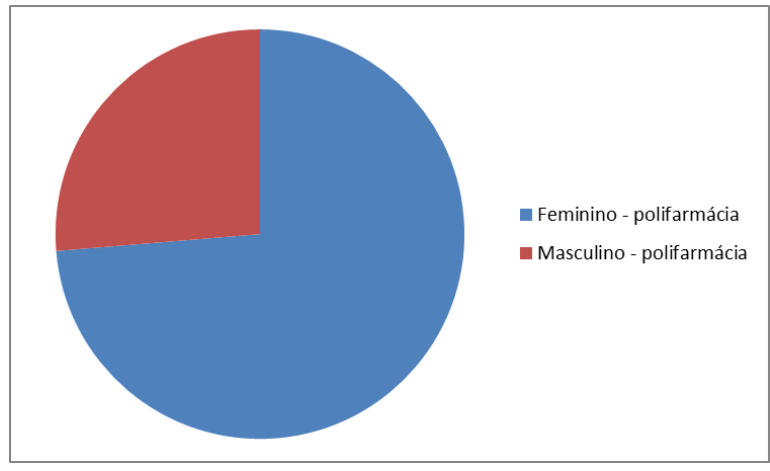

Fonte: autoria própria.
A pesquisa também investigou uma possível relação entre a prevalência da polifarmácia e a faixa etária dos idosos pesquisados. De acordo com os resultados, $31,09 \%$ dos idosos entre $60 \mathrm{e}$ 69 anos encontravam-se em polifarmácia, $46,49 \%$ dos idosos entre 70 e 79 anos estavam em polifarmácia e 48,97\% dos idosos entre 80 e 89 anos encontravam-se em polifarmácia. As análises permitem concluir que há uma relação significativa, onde quanto maior a faixa etária do idoso, maior o risco de polifarmácia. Resultados discordantes foram encontrados por Ramos et al., $(2016)^{9}$, onde a polifarmácia foi significativamente maior entre os idosos que se encontravam na faixa etária entre 70 a 79 anos. Aqui pode-se levantar a hipótese de que as características intrínsecas a cada população justifique tal discordância. Além disso, o $\mathrm{n}$ total da pesquisa, ainda que significativo para a cidade em questão, pode ser considerado pequeno para uma tentativa de generalização. Abaixo estão os gráficos que demonstram os resultados discutidos.

Gráfico 6: Quantidade de idosos x número de medicamentos. Patos de Minas (MG-Brasil), 2017.

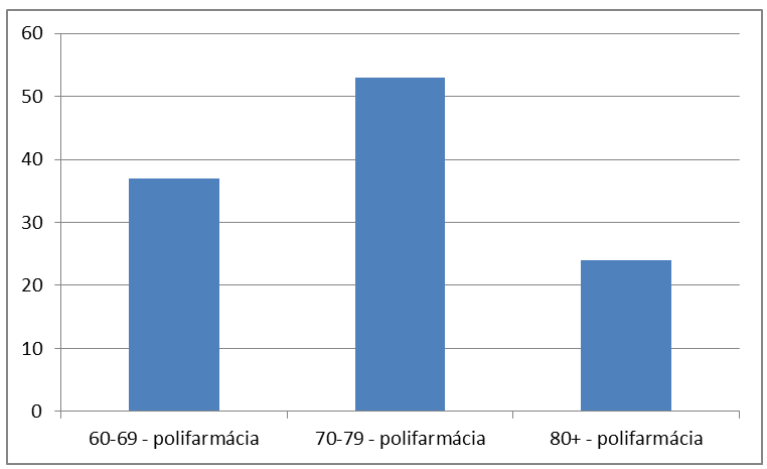

Fonte: autoria própria.

Outro dado interessante que foi obtido com a pesquisa foi o período da última consulta dos idosos pesquisados relacionado com a prevalência da polifarmácia. Dentre os idosos que consultaram entre o janeiro e junho de 2017, apenas 5,26\% encontram-se em polifarmácia. Dentre os idosos que consultaram de julho a dezembro de 2016, 59,6\% estavam utilizando polifarmácia. Já dentre os idosos que consultaram entre janeiro e junho de 2016, 35\% deles encontram-se em polifarmácia. Para esse dado não houve relação significativa, porém, é possível perceber o quanto foi baixa 
a porcentagem de idosos que se encontravam em polifarmácia e que tinham sido acompanhados recentemente. Aqui é possível levantar a hipótese de que esses idosos seriam aqueles que comparecem às consultas marcadas regularmente e, consequentemente, atingem um melhor controle de suas condições crônicas, diminuindo a necessidade de utilizar vários medicamentos.

Gráfico 7: Período de consulta x Polifarmácia. Patos de Minas (MG-Brasil), 2017.

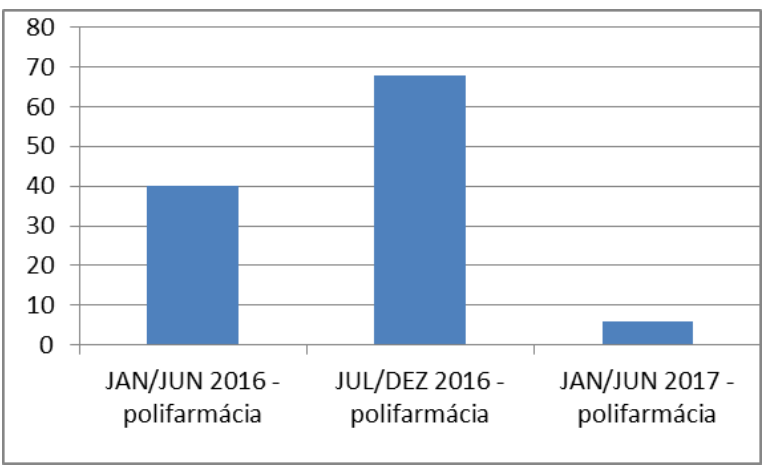

Fonte: autoria própria.

Esse acompanhamento regular e intervalos mais próximos entre as consultas são importantíssimos para os idosos, principalmente porque a maioria das condições crônicas que atingem essa faixa etária é controlada não só com medicamentos, mas com mudanças do estilo de vida como a realização de atividades físicas e dieta balanceada. Com um acompanhamento mais próximo e orientações médicas o paciente consegue criar metas e se motivar mais facilmente para atingi-las, melhorando cada vez mais sua saúde, e assim, reduzindo a necessidade de tomar medicamentos.

\section{CONCLUSÃO}

A prevalência da polifarmácia em idosos encontrada no presente estudo foi elevada, inclusive maior que os dados da literatura. Ficou evidente também a relação do gênero feminino com a polifarmácia, ou seja, ser idosa constituiu um fator de risco para polifarmácia, no presente estudo.

De acordo com o resultado obtido percebe-se também que houve uma relação significativa entre idade e polifarmácia, onde quanto maior a faixa etária do idoso, maior o risco de polifarmácia.

Chama atenção outro dado apresentado pela pesquisa, quando correlacionou o tempo entre a última consulta do idoso e a prevalência da polifarmácia. Foi muito baixa a porcentagem de idosos que se encontravam em polifarmácia e tinham consultas recentes, esse acompanhamento regular entre as consultas é fundamental para os idosos, propiciando um controle de suas condições crônicas e diminuindo a necessidade de utilizar vários medicamentos.

Persiste a possibilidade de a polifarmácia encontrada abranger também medicamentos inapropriados para idosos, nesse caso um assunto mais abrangente, uma vez que o uso de medicamento inapropriado é um dos principais fatores de risco para reações adversas em indivíduos idosos. Neste contexto, depreende-se que mais pesquisas deverão ser realizadas, abordando não somente a prevalência numérica da polifarmácia nos idosos, mas também citando suas características e relacionando com a lista dos critérios de Beers.

\section{REFERÊNCIAS}

1. Instituto Brasileiro de Geografia e Estatística - IBGE (Brasil): Atlas do Censo Demográfico 2010. Brasília, DF 2010. Bivar W. Bittencourt NDC.

2. Silva JOB, Gondim APS, Monteiro MP, Frota MA, Meneses ALL. Uso de medicamentos contínuos e fatores associados em idosos de Quixadá, Ceará. Rev. Bras. Epidemiol. 2012; 15(2): 386-95.

3. Silveira EA, Dalastra L, Pagotto V. Polifarmácia, doenças crônicas e marcadores nutricionais em idosos. Revista Brasileira de Epidemiologia. 2014; 17(4): 818-9.

4. Carvalho MFC. A polifarmácia em idosos do Município de São Paulo: Estudo SABE - Saúde, Bem-estar e Envelhecimento. São Paulo. Dissertação [Mestrado em Saúde Pública]. Faculdade de Saúde Pública Universidade de São Paulo, 2007.

5. Freitas IV, PY L. Tratado de Geriatria e Gerontologia. $3^{\text {ed }}$. Rio de Janeiro: Guanabara Koogan; 2011

6. Gomes HO, Caldas CP. Uso inapropriado de medicamentos pelo idoso: polifarmácia e seus efeitos. Revista Hospital Universitário Pedro Hernesto. 2008; 1: 1-5.

7. Costa EF, Porto CC, Soares AT. Envelhecimento populacional brasileiro e o aprendizado de geriatria e gerontologia. Revista da Universidade Federal de Goiás. 2003; 1(1): 12-16. 
8. Prybys KM, Melville KA, Frederick P. Hanna JR. Polypharmacy in the elderly: clinical challenges in emergency practice: Part 1 overview, etiology, and drug interactions. Emerg Med Rep. 2002; 23(8):145-53.

9. Ramos LR, Tavares NUL, Bertoldi AD, Farias MR, Oliveira MA, Luiza VL, Pizzol TS, Arrais SSD, Mengue SS. Polifarmácia e polimorbidade em idosos no Brasil: um desafio em saúde pública. Revista de Saúde Pública. 2016; 50(Sup2): 9.

10. Almeida NA, Reiners AAO, Azevedo RCS, Silva AMC, Cardoso JDC, Souza LC. Prevalência e fatores associados à polifarmácia entre os idosos residentes na comunidade. Revista Brasileira de Geriatria e Gerontologia. 2017; 20(1): 138-48. 